\section{E-Mail-Newsletter: Service und Information für Fachärzte zu Krebs}

Der E-Mail-Newsletter «Onkologie im Fokus» der Roche Pharma AG informiert Fachärzte über aktuelle Entwicklungen und Themen in der onkologischen Forschung und Praxis.

Aktuelle Forschungsergebnisse, innovative Therapieoptionen, wichtige Fortbildungsangebote: Stets auf dem neuesten Stand in Forschung und Wissenschaft zu bleiben, ist für Ärzte eine ständige Herausforderung. Präzise, übersichtlich und fundiert recherchiert informiert der jetzt verfügbare E-Mail-Newsletter der Roche Pharma AG Fachärzte zum
Thema Onkologie. Große Fortschritte in der Erforschung und Therapie von Krebserkrankungen wurden in den vergangenen Jahren erzielt. Der quartalsweise erscheinende EMail-Newsletter bietet neben interessant aufbereiteten Kongressberichten, Studiendaten und Analysen auch Hintergrundinformationen zu neuen Therapieoptionen. Außerdem enthält der E-Mail-Newsletter eine Übersicht $\mathrm{zu}$ wichtigen Kongressterminen und interessanten zertifizierten Fortbildungen (CME).

Dieser Service ist für die Abonnenten kostefrei und kann online unter

www.roche.de/pharma/newsletter

oder unter www.onkologie-im-fokus.de

bestellt werden.
Der E-Mail-Newsletter «Onkologie im Fokus» steht als Text- oder HTML-Mail sowie als PDF-Dokument zur Verfügung.

Weitere Informationen bei

Annegret Meier

medical relations $\mathrm{GmbH}$

Hans-Böckler-Str. 46

40764 Langenfeld

Tel. + 492173 9769-56, Fax -49

annegret.meier@medical-relations.de

\section{Hohe Ansprechrate unter Caspo- fungin in der Routineversorgung der invasiven Aspergillose}

In einer prospektiven Beobachtungsstudie hat die CAN-DO(IA)-Studiengruppe zwischen April 2006 und September 2007 Patienten erfasst, die eine nachgewiesene oder wahrscheinliche invasive Aspergillose hatten und in der klinischen Routineversorgung mit Caspofungin behandelt worden waren. Im Unterschied zu randomisierten Studien wurde die Aufnahme von Patienten - abgesehen von einem Alter $>16$ Jahre - nicht durch weitere Ausschlusskriterien eingeschränkt.

Von insgesamt 103 aufgenommenen Patienten aus 11 Ländern konnte bei 101 das Ergebnis bewertet werden. Häufigste Grunderkrankungen waren Malignom (77\%) und Zustand nach allogener Stammzelltranstransplantation (22\%). 57\% waren zu Beginn der Therapie noch neutropenisch. Die Aspergillose war in $30 \%$ der Fälle nachgewiesen und bei $70 \%$ wahrscheinlich, gemäß den modifizierten Kriterien der EORTC ${ }^{\mathrm{a}}$-Mycoses Study Group. Häufigste Lokalisation war die Lunge (84\%).
Caspofungin wurde 20 Patienten als Ersttherapie $^{\mathrm{b}}$ und 83 Patienten als Salvagetherapie verabreicht, vor allem nach Versagen der Vorbehandlung (Amphotericin B, Fluco-, Itra- oder Voriconazol sowie liposomales Amphotericin B), und ganz überwiegend als Monotherapie (83\%) und in Standarddosierung (92\%).

Die Ansprechrate (vollständiges und teilweises Ansprechen) betrug 56\% sowohl im Gesamtkollektiv als auch in den meisten Subgruppen (Mono- und Kombinationstherapie, Salvagetherapie, nachgewiesene und wahrscheinliche Aspergillose), bei neutropenischen Patienten 53\% und bei Ersttherapie sogar 60\%. Die Gesamtüberlebensrate bei Therapieende war $73 \%$.

Die Behandlung wurde von den meisten Patienten gut vertragen. $\mathrm{Zu}$ ernsten klinischen $\mathrm{Ne}$ benwirkungen kam es nur bei drei (2,9\%) Patienten, von denen zwei die Behandlung abbrachen. Kein Patient musste die Therapie aufgrund von Arzneimittelinteraktionen abbrechen.
Quelle

J. Maertens, G. Egerer, W. Shin, D. Reichert, M. Stek, S. Chandwani, M. Shivaprakash, C. Viscoli for the CAN-DO(IA) study team: Caspofungin use in daily clinical practice for treatment of invasive aspergillosis - results of a prospective observational registry. 18th European Congress of Clinical Microbiology and Infectious Diseases (ECCMID), Poster 1017.

${ }^{\mathrm{a} E O R T C}=$ European Organisation for Research and Treatment of Cancer

${ }^{\mathrm{b}} \mathrm{CANCIDAS}^{\circledR}$ ist in Deutschland bei invasiver Aspergillose nicht als Ersttherapie zugelassen.

Weitere Informationen bei

MSD SHARP und DOHME GMBH

Fulvia Kipper

Lindenplatz 1

85540 Haar

Tel. +49 89 4561-1917, Fax -1329

fulvia_kipper@msd.de

\title{
PharmaTicker+++ PharmaTicker+++ PharmaTicker+++ PharmaTicker+++
}

Die Deutsche Krebsgesellschaft e.V. hat gemeinsam mit anderen medizinischen Fachgesellschaften, Verbänden und Arbeitsgemeinschaften die interdisziplinäre S3-Leitlinie für die Diagnostik, Therapie und Nachsorge des Mammakarzinoms überarbeitet und an neue $\mathrm{Er}$ kenntnisse angepasst. Unter www.krebsgesellschaft.de kann die aktualisierte Leitlinie ab sofort abgerufen werden.

Weitere Informationen bei

Pressestelle der Deutschen Krebsgesellschaft e.V.

André Franck

Tel. +49 3643 743-749, Fax -536

presse@krebsgesellschaft.de
Janssen-Cilag GmbH. Für viele Patienten mit starken chronischen Schmerzen eignet sich ein niedrig dosiertes Opioid der Stufe III bereits als Einstieg in die Schmerztherapie. Insbesondere Hydromorphon in der $\mathrm{OROS}^{\circledR}$-Technologie, wie Jurnista $^{\circledR}$, ermöglicht durch eine kontinuierliche Wirkstofffreisetzung gleichmäßige Plasmaspiegel und so eine gleichmäßige Analgesie.

PUBLICIS VITAL PR

Kommunikation für Gesundheit $\mathrm{GmbH}$

Kerstin Krätsch

Hansaring 97, 50670 Köln

Tel. +49 221 912719-32

kerstin.kraetsch@publicis-vital-pr.de
Lilly Deutschland GmbH. Ärztliche Therapiefreiheit ist kein überflüssiger Luxus, sondern eine notwendige Voraussetzung für die von $\mathrm{Pa}$ tienten geforderte Individualisierung der Therapie. Dies ist das Fazit einer gesundheitspolitischen Dialogveranstaltung, die am 25./26. Januar 2008 in Berlin stattfand. Die Veranstaltung kann als Medical Mobile Lecture unter www. lilly-pharma.de «Dialog im Gesundheitswesen» angesehen und heruntergeladen werden.

3K Agentur für Kommunikation $\mathrm{GmbH}$

Sabine Theobald

Tel. +49 69 971711-0, Fax -22

info@3k-komm.de 


\section{Risikoadaptierte Behandlung des metastasierten HRPC: Cave: Alter per se ist kein Risikofaktor!}

Metastasierte Patienten mit hormonrefraktärem Prostatakarzinom (HRPC) benötigen eine individuelle, risikoadaptierte Behandlung. Die Risikoeinteilung orientiert sich an Komorbiditäten bzw. dem allgemeinen Gesundheitszustand. Kein Risikofaktor per se ist das erhöhte Lebensalter ( $>70$ Jahre). Ältere Patienten mit metastasiertem HRPC profitieren grundsätzlich im gleichen Umfang von der 3-wöchentlichen Chemotherapie mit Docetaxel/Prednison wie jüngere Patienten. Bei deutlichen Einschränkungen bietet sich eine adaptierte Chemotherapie, z.B. die wöchentliche Gabe von Docetaxel/ Prednison an, erläuterte Prof. Jean-Pierre Droz, Lyon/Frankreich, anlässlich des diesjährigen EAU-Kongresses.
Komorbiditäten und Gesundheitszustand lassen sich anhand des Charlson Comorbidity Index und diversen Nomogrammen, z.B. jenem nach d'Amico validieren. Von entscheidender Bedeutung ist hierbei die klinische Untersuchung. Sie sollte interdisziplinär erfolgen, da auch der Ernährungszustand, die kognitive Verfassung des Patienten sowie Pflegebedürftigkeit und Komedikationen eine Rolle spielen. Unterschieden werden vier Gruppen: (1) der - abgesehen vom Prostatakarzinom - gesunde ältere Patient («fitter» Patient), (2) der ältere Patient mit leichten, reversiblen bzw. behandelbaren Einschränkungen («vulnerabler» Patient), (3) der ältere Patient mit klinisch relevanten, irreversiblen Komorbiditäten («frail» = deutlich geschwächter Patient) und (4) der nur noch «palliativ» zu behandelnde Patient.

Die Patienten der Gruppe 1 und 2 profitieren laut Droz von der effektiven Standardtherapie genauso wie jüngere Patienten. Standard für Patienten mit metastasiertem HRPC ist die 3wöchentliche Chemotherapie mit Docetaxel (75 $\mathrm{mg} / \mathrm{m}^{2}$ ) plus niedrig dosiertes Prednison. Patienten der Gruppe 3 mit klinisch relevanten, irreversiblen Einschränkungen sollten eine adaptierte Chemotherapie erhalten. Eine valide Option ist laut Droz die wöchentliche Gabe von 30 $\mathrm{mg} / \mathrm{m}^{2}$ Docetaxel plus Prednison.

\section{Weitere Informationen bei}

Cristina Iannazzo

Sanofi-Aventis Deutschland $\mathrm{GmbH}$ Tel. +49 69 305-24417, Fax -188 02 presse@sanofi-aventis.com

\section{Follikuläres Lymphom: EU-Zulassung für Zevalin ${ }^{\circledR}$ als Erst- linien-Konsolidierungstherapie}

Die Europäische Kommission hat die Zulassung für Zevalin ${ }^{\circledR}$ ([90Y]-Ibritumomab-Tiuxe$\tan )$ in Europa erweitert. Damit kann Zevalin nun auch im Rahmen der Erstbehandlung bei Patienten mit follikulärem Lymphom eingesetzt werden, die auf eine Anfangstherapie angesprochen haben. Ziel einer solchen Konsolidierungstherapie ist die Verbesserung der Wirkung einer ersten Initialbehandlung. Der therapeutische Nutzen von Zevalin nach einer Behandlung mit Rituximab in Kombination mit einer Chemotherapie ist nicht ermittelt. Zevalin wurde in Europa erstmalig 2004 zugelassen. Es verbindet die zielgenaue Bindungsfähigkeit eines monoklonalen Anti-CD20-Antikörpers mit den tumorzerstörenden Eigenschaften einer lokalisierten Strahlentherapie mit Yttrium-90. Das follikuläre Lymphom ist eine der häufigsten Formen des Non-Hodgkin-Lymphoms, einem Tumor des Lymphsystems.

Die Entscheidung der Europäischen Kommission zur Erteilung der Zulassungserweiterung für Zevalin stützt sich auf Daten aus der Phase-IIIStudie «First-Line Indolent Trial» (FIT-Studie). Darin konnte gezeigt werden, dass Zevalin als Erstlinien-Konsolidierungstherapie die mittlere progressionsfreie Überlebenszeit signifikant von 13,5 Monaten (Kontrollarm) auf 37 Monate verlängerte $(\mathrm{p}<0,0001)$.

Die FIT-Studie (First-Line Indolent Trial) ist eine multinationale, randomisierte Phase-IIIStudie zur Untersuchung von Zevalin als Erstlinien-Konsolidierungstherapie, verabreicht als therapeutische Einzeldosis an Patienten mit fortgeschrittenem follikulärem Lymphom (Stadium III oder IV), die nach den Standard-Erstli-
nien-Chemotherapieregimes eine Teilremission oder vollständige Remission erreicht hatten. Ziel der FIT-Studie ist die Beurteilung des Nutzens und der Sicherheit einer Konsolidierungstherapie mit Zevalin nach Erstlinientherapie bei Patienten mit follikulärem Lymphom, einer der häufigsten Formen des Non-Hodgkin-Lymphoms. Die Bayer Schering Pharma AG besitzt die weltweiten exklusiven Vermarktungsrechte für Zevalin mit Ausnahme der USA.

Weitere Informationen bei Bayer Schering Pharma AG

Anna Schuberth

Tel. +49 30 468-15942

anna.schuberth@bayerhealthcare.com

\section{PharmaTicker+++ PharmaTicker+++ PharmaTicker+++ PharmaTicker+++}

Ortho Biotech, Division of Janssen-Cilag GmbH. Beim diesjährigen Deutschen Krebskongress war der Proteasom-Inhibitor Bortezomib (Velcade ${ }^{\circledR}$ ) ein zentrales Thema der wissenschaftlichen Diskussion: Der als Monosubstanz ab dem ersten Rezidiv, nach erfolgter oder nicht angezeigter Stammzelltransplantation zugelassene Wirkstoff ermöglicht eine hohe Ansprechqualität und kann auch unter Praxisbedingungen sowie bei Niereninsuffizienz medizinisch überwacht von Nutzen sein.

SanCom Creative Communication Solutions $\mathrm{GmbH}$

Yasmin König

Tel. +49 6123 70558-35

koenig@sancom-ccs.de
Roche Pharma AG. Im Kampf gegen Knochenmetastasen senken Bisphosphonate drastisch die Inzidenz hyperkalzämischer Episoden und reduzieren die Zahl skelettaler Komplikationen. Vorallem aber reduzieren sie Knochenschmerzen und verbessern so die Lebensqualiät der Patienten. Neue Erkenntnis zu dieser mittlerweile sehr gut erforschten Substanzklasse brachte der Deutsche Krebskongress 2008.

Weitere Informationen bei

Dr. H.-U. Jelitto

Roche Pharma AG

Tel. +49 7624 14-3715, Fax -3366

www.roche.de
Cephalon GmbH. Über die Hälfte aller Tumorpatienten mit starken chronischen Schmerzen haben trotz adäquater Opioid-Basismedikation regelmäßig Durchbruchschmerzen, was die Lebensqualität erheblich beeinträchtigt. Dennoch werden Durchbruchschmerzen bei der Versorgung immer noch zu wenig beachtet. Durch schnell wirkende Opioide wie oral-transmukosales Fentanylcitrat $\left(\right.$ Actiq $\left.^{\circledR}\right)$ können Schmerzspitzen gut kontrolliert werden.

Pleon GmbH

Eszter Viragh

Tel. +49 89 59042-1227, Fax -1100 eszter.viragh@pleon.com 